\title{
Depth Camera Based Indoor Mobile Robot Localization and Navigation
}

\author{
Joydeep Biswas \\ The Robotics Institute \\ Carnegie Mellon University \\ Pittsburgh, PA 15213, USA \\ joydeepberi. cmu . edu
}

\author{
Manuela Veloso \\ Computer Science Department \\ Carnegie Mellon University \\ Pittsburgh, PA 15213, USA \\ mmvecs. cmu . edu
}

\begin{abstract}
The sheer volume of data generated by depth cameras provides a challenge to process in real time, in particular when used for indoor mobile robot localization and navigation. We introduce the Fast Sampling Plane Filtering (FSPF) algorithm to reduce the volume of the 3D point cloud by sampling points from the depth image, and classifying local grouped sets of points as belonging to planes in 3D (the "plane filtered" points) or points that do not correspond to planes within a specified error margin (the "outlier" points). We then introduce a localization algorithm based on an observation model that down-projects the plane filtered points on to $2 \mathrm{D}$, and assigns correspondences for each point to lines in the 2D map. The full sampled point cloud (consisting of both plane filtered as well as outlier points) is processed for obstacle avoidance for autonomous navigation. All our algorithms process only the depth information, and do not require additional RGB data. The FSPF, localization and obstacle avoidance algorithms run in real time at full camera frame rates $(30 \mathrm{~Hz})$ with low CPU requirements $(16 \%)$. We provide experimental results demonstrating the effectiveness of our approach for indoor mobile robot localization and navigation. We further compare the accuracy and robustness in localization using depth cameras with FSPF vs. alternative approaches that simulate laser rangefinder scans from the $3 D$ data.
\end{abstract}

\section{INTRODUCTION}

The recent availability of inexpensive depth cameras has made available dense 3D point clouds, which were previously only accessible using much more expensive sensors like time-of-flight cameras or scanning 3D laser rangefinders. We are interested in using these depth cameras for ground based indoor mobile robots. We consider mobile robots with limited onboard computational power, and address two immediate challenges to using the depth cameras for mobile robot localization and navigation:

1) Depth cameras typically generate voluminous data that cannot be processed in its entirety in real time for localization (e.g., the Microsoft Kinect sensor produces 9.2 million 3D pts/sec, compared to the $68002 \mathrm{D}$ pts/sec of the Hokuyo URG-04lx laser rangefinder).

2) Given that we already have existing $2 \mathrm{D}$ maps of our indoor environments, the observed 3D point clouds should be matched with the 2D maps.

In this paper, we tackle both these challenges. We first introduce the Fast Sampling Plane Filtering (FSPF) algorithm that samples the depth image to produce a set of points corresponding to planes, along with the plane parameters (normals and offsets). The volume of data to be processed is

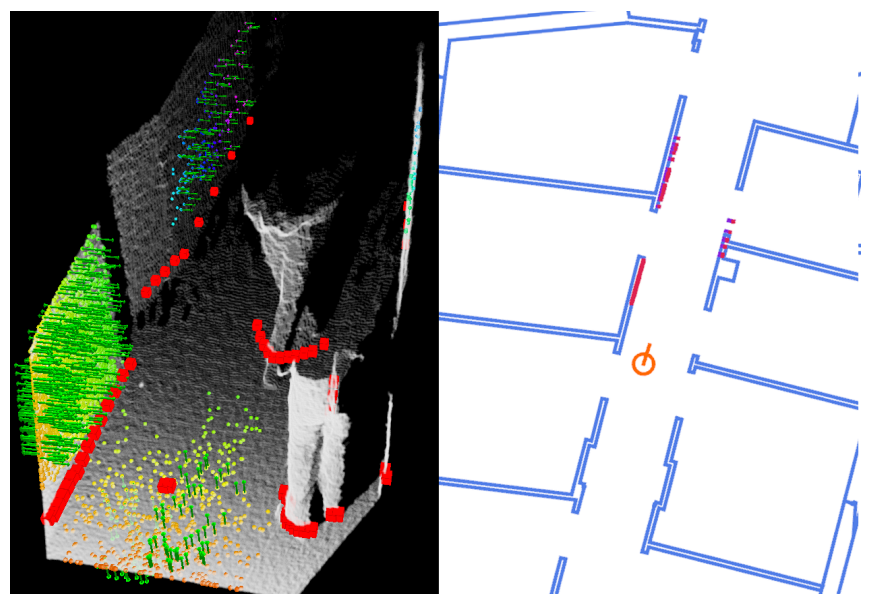

Fig. 1. Snapshot of depth image processing: On the left, the complete 3D point cloud is shown in white, the plane filtered 3D points in color along with plane normals, and the obstacle avoidance margins denoted by red boxes. On the right, the robot's pose is shown on the vector map (blue lines), with the 3D point correspondences shown as red points.

thus significantly reduced, addressing the first challenge with the additional advantage that non-planar objects in the scene, which are unlikely to correspond to map features are filtered. We then address the second challenge by introducing an observation model in our localization algorithm that matches the plane filtered points to the lines in the 2D maps, making it possible to reuse our existing 2D maps. This is in contrast to, and more effective than (as we shall show) down-projecting the 3D point cloud and binning it to simulate a conventional laser rangefinder. Our combined approach interestingly uses only the depth image and does not require the RGB images. The sampled points generated are also used to perform obstacle avoidance for navigation of the robot. Fig. 1 shows a snapshot of the key processed results after plane filtering, localization, and computing the obstacle avoidance margins.

Since our application is based on a ground robot, 6 degree of freedom (6D) localization is not required since the height of the sensor on the robot and its tilt and roll angles are fixed. At the same time, observations in 3D made by the depth camera have the potential to provide more useful information than planar laser rangefinders that sense objects only on a single 2D plane. Furthermore, typical indoor environments have an abundance of large planar features that are discernible in the depth images. 


\section{RELATED WORK}

Approaches that operate on raw 3D point clouds for plane (and general geometric shape) detection [1], [2] are, without modifications, ill-suited to running in real-time on depth images due to their high computational requirements, and because they ignore the fact that depth cameras make observations in "2.5D": the depth values are observed on a (virtual) 2D image plane originating from a single point. Region growing [3] exploits the local correlation in the depth image and attempts to assign planes to every $3 \mathrm{D}$ point. The Fast Sampling Plane Filtering algorithm, which we introduce in this paper, in contrast samples points at random and does not attempt to fit planes to every point, and instead uses local RANSAC [4].

There has been considerable work in 2D localization and mapping ([5] provides an overview of the recent advances in SLAM), and in using localization on 2D maps to generate 3D models using additional scans [6]. Specific to the problem of building 3D maps with 6 degrees of freedom (6D) localization is 6D SLAM [7], [8] that builds maps using $3 \mathrm{D}$ points in space, but these methods do not reason about the geometric primitives that the $3 \mathrm{D}$ points approximate.

An alternative approach to mapping using the raw 3D points is to map using planar features extracted from the 3D point cloud [9], [10]. In particular, 3D Plane SLAM [11] is a 6D SLAM algorithm that uses observed 3D point clouds to construct maps with 3D planes. The plane detection in their work relies on region growing [3] for plane extraction, whereas our approach uses sampling of the depth image. In addition, our observation model projects the observed planes onto the existing $2 \mathrm{D}$ vector map used for $2 \mathrm{D}$ laser rangefinder sensors.

More recently, techniques for 6D localization and mapping using RGB-D cameras have been explored. One such approach constructs surface element based dense 3D maps [12] that are simultaneously used for localizing in 3D using iterative closest point (ICP) as well as visual feature (SIFT) matching. While such approaches generate visually appealing dense 3D maps, they include in the maps features resulting from objects that are not likely to persist over time, like objects placed on tables, and the locations of movable chairs and tables.

In summary, the main contributions of this paper in relation to other work are:

- The Fast Sampling Plane Filtering algorithm that samples the depth image to produce a set of points corresponding to planes (Section III)

- A localization algorithm that uses this filtered point cloud to localize on a 2D vector map (Section IV)

- An obstacle avoidance algorithm that enables safe autonomous navigation (Section $\mathrm{V}$ )

- Experimental results (Section VI) showing the accuracy and reliability of FSPF based localization compared to the approach of localizing using simulated laser rangefinder scans, and long run autonomous trials of the robot using the depth camera alone.

\section{Fast Sampling Plane Filtering}

Depth cameras provide, for every pixel, color and depth values. This depth information, along with the camera intrinsics (horizontal field of view $f_{h}$, vertical field of view $f_{v}$, image width $w$ and height $h$ in pixels) can be used to reconstruct a 3D point cloud. Let the depth image of size $w \times h$ pixels provided by the camera be $I$, where $I(i, j)$ is the depth of a pixel at location $d=(i, j)$. The corresponding 3D point $p=\left(p_{x}, p_{y}, p_{z}\right)$ is reconstructed using the depth value $I(d)$ as $p_{x}=I(d)\left(\frac{j}{w-1}-0.5\right) \tan \left(\frac{f_{h}}{2}\right), p_{y}=$ $I(d)\left(\frac{i}{h-1}-0.5\right) \tan \left(\frac{f_{v}}{2}\right), p_{z}=I(d)$.

FSPF takes the depth image $I$ as its input, and creates a list $P$ of $n$ 3D points, a list $R$ of corresponding plane normals, and a list $O$ of outlier points that do not correspond to any planes. Algorithm 1 outlines the plane filtering procedure. It uses the helper subroutine [numInliers, $\hat{P}, \hat{R}] \leftarrow \operatorname{RANSAC}\left(d_{0}, w^{\prime}, h^{\prime}, l, \epsilon\right)$, which performs the classical RANSAC algorithm over the window of size $w^{\prime} \times h^{\prime}$ around location $d_{0}$ in the depth image, and returns inlier points and normals $\hat{P}$ and $\hat{R}$ respectively, as well as the number of inlier points found. The configuration parameters required by FSPF are listed in Table I.

\begin{tabular}{|l|l|l|}
\hline Parameter & Value & Description \\
\hline$n_{\max }$ & 2000 & Maximum total number of filtered points \\
$k_{\max }$ & 20000 & Maximum number of neighborhoods to sample \\
$l$ & 80 & Number of local samples \\
$\eta$ & 60 & Neighborhood for global samples (in pixels) \\
$S$ & $0.5 \mathrm{~m}$ & Plane size in world space for local samples \\
$\epsilon$ & $0.02 \mathrm{~m}$ & Maximum plane offset error for inliers \\
$\alpha_{i n}$ & 0.8 & Minimum inlier fraction to accept local sample \\
\hline
\end{tabular}

TABLE I

CONFIGURATION PARAMETERS FOR FSPF

FSPF proceeds by first sampling three locations $d_{0}, d_{1}, d_{2}$ from the depth image (lines 9-11). The first location $d_{0}$ is selected randomly from anywhere in the image, and then $d_{1}$ and $d_{2}$ are selected from a neighborhood of size $\eta$ around $d_{0}$. The 3D coordinates for the corresponding points $p_{0}, p_{1}$, $p_{2}$ are then computed (line 12). A search window of width $w^{\prime}$ and height $h^{\prime}$ is computed based on the mean depth ( $z$ coordinate) of the points $p_{0}, p_{1}, p_{2}$ (lines 14-16) and the minimum expected size $S$ of the planes in the world. Local RANSAC is then performed in the search window. If more than $\alpha_{i n} l$ inlier points are produced as a result of running RANSAC in the search window, then all the inlier points are added to the list $P$, and the associated normals (computed using a least-squares fit on the RANSAC inlier points) to the list $R$. This algorithm is run a maximum of $m_{\max }$ times to generate a list of maximum $n_{\max } 3 \mathrm{D}$ points and their corresponding plane normals. Fig. 2 shows an example scene with the plane filtered points and their corresponding plane normals.

\section{LOCALIZATION}

For the task of localization, the plane filtered point cloud $P$ and the corresponding plane normal estimates $R$ need to be related to the $2 \mathrm{D}$ map. The $2 \mathrm{D}$ map representation that 


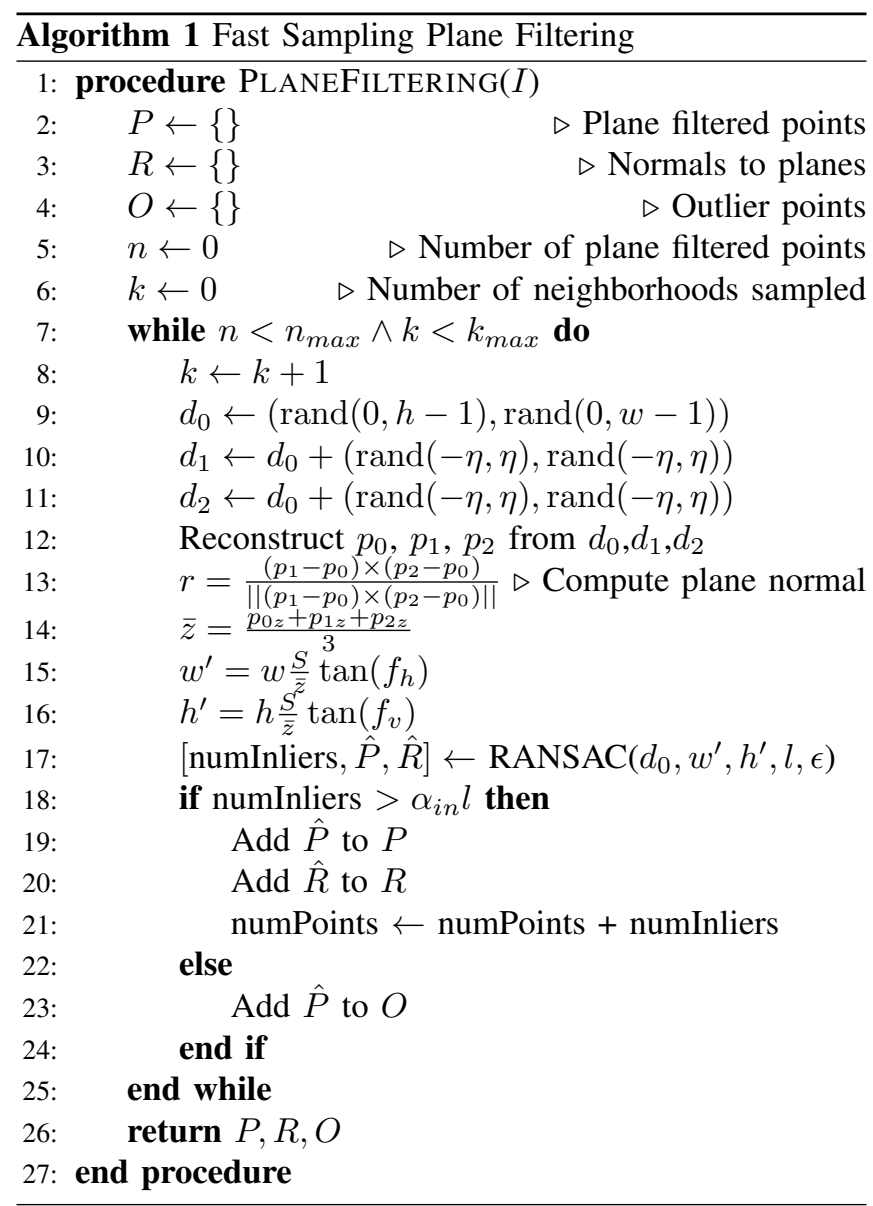

we use is a "vector" map: it represents the environment as a set of line segments (corresponding to the obstacles in the environment), as opposed to the more commonly used occupancy grid [13] based maps. The observation model therefore has to compute the line segments likely to be observed by the robot given its current pose and the map. This is done by an analytic ray cast step. We therefore introduce next the representation of the 2D vector map and the algorithm for analytic ray casting using the $2 \mathrm{D}$ vector map.

\section{A. Vector Map Representation and Analytic Ray Casting}

The map $M$ used by our localization algorithm is a set of $s$ line segments $l_{i}$ corresponding to all the walls in the environment: $M=\left\{l_{i}\right\}_{i=1: s}$. Such a representation may be acquired by mapping (e.g. [14]) or (as in our case) taken from the blueprints of the building.

Given this map, to compute the observation likelihoods based on observed planes, the first step is to estimate which lines on the map are likely to be observed (the "scene lines"), given the pose estimate of the robot. This ray casting step is analytically computed using the vector map representation.

The procedure to analytically generate a ray cast at location $x$ given the map $M$ is outlined in Algorithm 2 . The returned result is the scene lines $L$ : a list of nonintersecting, non-occluded line segments visible by the robot

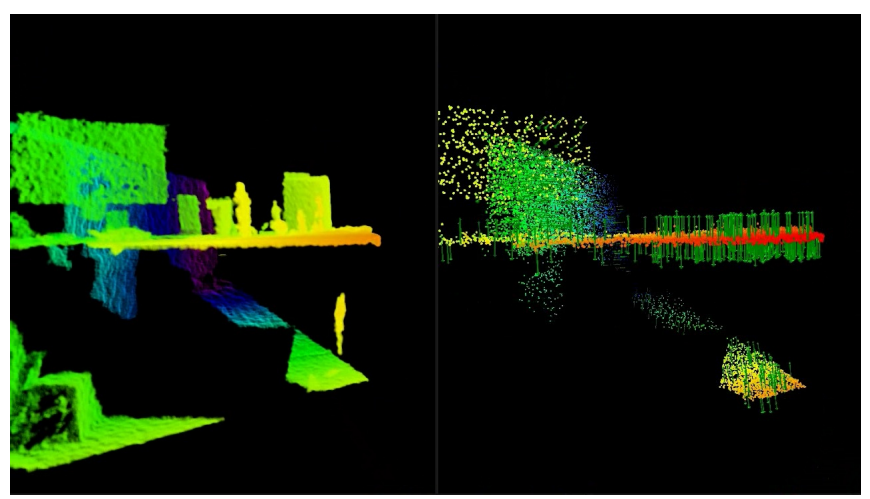

Fig. 2. Fast Sampling Plane Filtering in a scene with a cluttered desktop. The complete 3D point cloud is shown on the left, the plane filtered points and the corresponding normals on the right. The table clutter is rejected by FSPF while preserving the large planar elements like the monitors, the table surface, the walls and the floor.

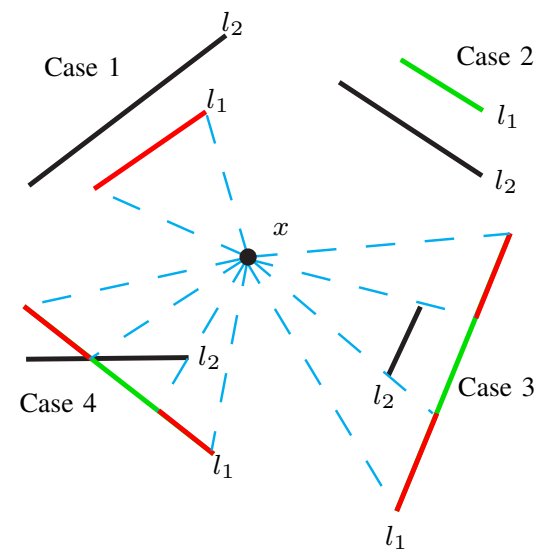

Fig. 3. Line occlusion cases. Line $l_{1}$ is being tested for occlusion by line $l_{2}$ from location $x$. The occluded parts of $l_{1}$ are shown in green, and the visible parts in red. The visible ranges are bounded by the angles demarcated by the blue dashed lines.

from the location $x$. This algorithm calls the helper procedure TrimOcclusion $\left(x, l_{1}, l_{2}, L\right)$ that accepts a location $x$, two lines $l_{1}$ and $l_{2}$ and a list of lines $L$. TrimOcclusion trims line $l_{1}$ based on the occlusions due to the line $l_{2}$ as seen from the location $x$. The list $L$ contains lines that yet need to be tested for occlusions by $l_{2}$. There are in general 4 types of arrangements of $l_{1}$ and $l_{2}$, as shown in Fig. 3:

1) $l_{1}$ is not occluded by $l_{2}$. In this case, $l_{1}$ is unchanged.

2) $l_{1}$ is completely occluded by $l_{2} . l_{1}$ is trimmed to zero length by TrimOcclusion.

3) $l_{1}$ is partially occluded by $l_{2} . l_{1}$ is first trimmed to a non occluded length, and if a second disconnected non occluded section of $l_{1}$ exists, it is added to $L$.

4) $l_{1}$ intersects with $l_{2}$. Again, $l_{1}$ is first trimmed to an non occluded length, and if a second disconnected non occluded section of $l_{1}$ exists, it is added to $L$.

The analytic ray casting algorithm (Algorithm 2) proceeds as follows: A list $\hat{L}$ of all possible lines is made from the map $M$. Every line $l_{i} \in \hat{L}$ is first trimmed based on occlusions by lines in the existing scene list $L$ (lines 5-7). If at least part of $l_{1}$ is left non occluded, then the existing lines in $\hat{L}$ are trimmed based on occlusions by $l_{i}$ (lines 9-11) and $l_{i}$ is 

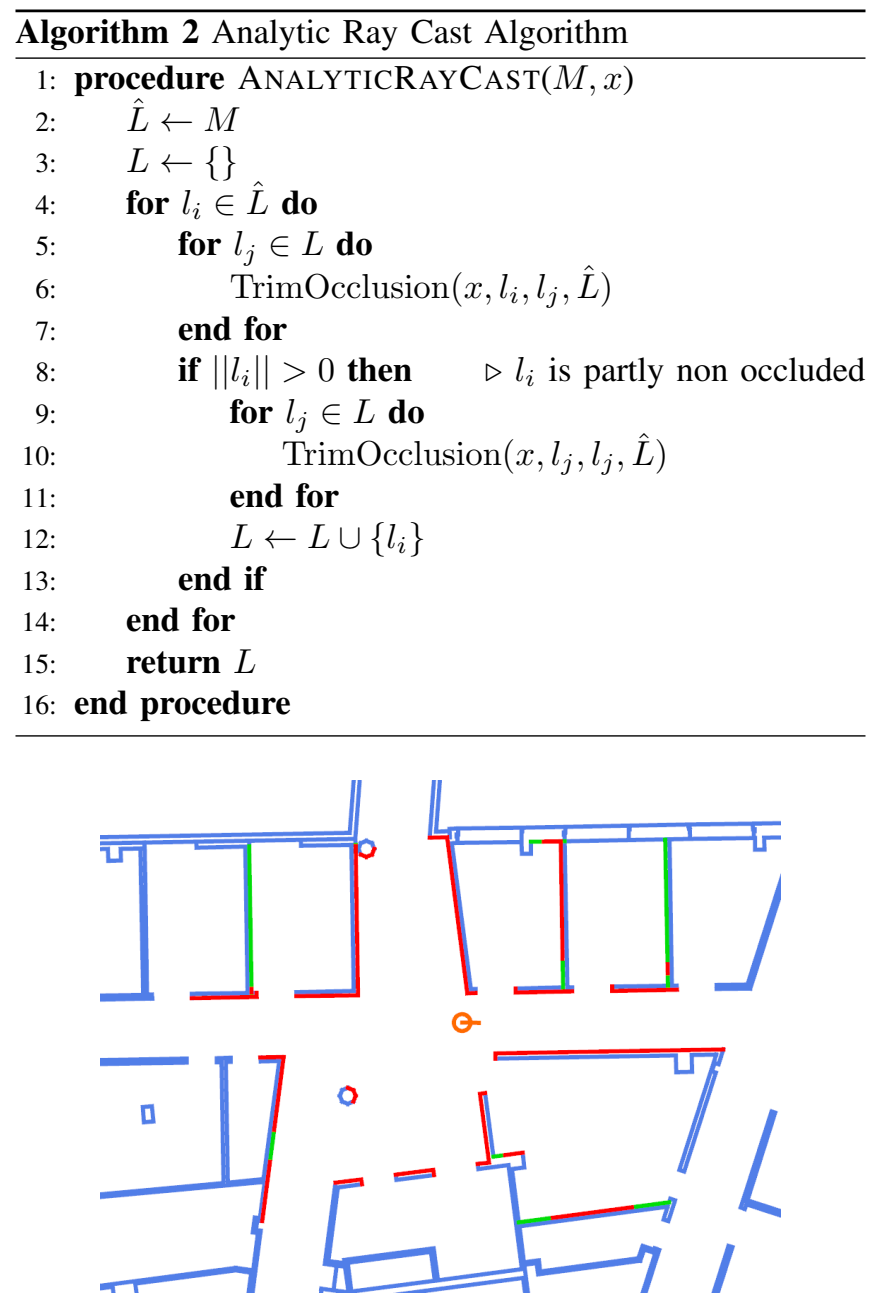

Fig. 4. Analytically rendered scene list. The lines in the final scene list $L$ are shown in red, the original, untrimmed corresponding lines in green, and all other lines on the map in blue.

then added to the scene list $L$. The result is a list of non occluded, non-intersecting scene lines in L. Fig. 4 shows an example scene list on the real map.

Thus, given the robot pose, the set of line segments likely to be observed by the robot is computed. Based on this list of line segments, the actual observation of the plane filtered point cloud $P$ is related to the map using the projected 3D point cloud model, which we introduce next.

\section{B. Projected 3D Point Cloud Observation Model}

Since the map on which the robot is localizing is in 2D, the 3D filtered point cloud $P$ and the corresponding plane normals $R$ are first projected onto 2D to generate a 2D point cloud $P^{\prime}$ along with the corresponding normalized normals $R^{\prime}$. Points that correspond to ground plane detections are rejected at this step. Let the pose of the robot $x$ be given by $x=\left\{x_{1}, x_{2}\right\}$ where $x_{1}$ is the 2D location of the robot, and $x_{2}$ its orientation angle. The observable scene lines list $L$ is computed using an analytic ray cast. The observation likelihood $p(y \mid x)$ (where the observation $y$ is the $2 \mathrm{D}$ projected point cloud $P^{\prime}$ ) is computed as follows:

1) For every point $p_{i}$ in $P^{\prime}$, line $l_{i}\left(l_{i} \in L\right)$ is found such that the ray in the direction of $p_{i}-x_{1}$ and originating from $x_{1}$ intersects $l_{i}$.

2) Points for which no such line $l_{i}$ can be found are discarded.

3) Points $p_{i}$ for which the corresponding normal estimates $r_{i}$ differ from the normal to the line $l_{i}$ by a value greater than a threshold $\theta_{\max }$ are discarded.

4) The perpendicular distance $d_{i}$ of $p_{i}$ from the (extended) line $l_{i}$ is computed.

5) The total (non-normalized) observation likelihood $p(y \mid x)$ is then given by:

$$
p(y \mid x)=\prod_{i=1}^{n} \exp \left[-\frac{d_{i}^{2}}{2 f \sigma^{2}}\right]
$$

Here, $\sigma$ is the standard deviation of a single distance measurement, and $f: f>1$ is a discounting factor to discount for the correlation between rays. The observation likelihoods thus computed are used for localization using the Corrective Gradient Refinement (CGR) [15] algorithm, which we review in brief.

\section{Corrective Gradient Refinement for Localization}

The belief of the robot's location is represented as a set of weighted samples or "particles", as in Monte Carlo Localization (MCL)[16]: $\operatorname{Bel}\left(x_{t}\right)=\left\{x_{t}^{i}, w_{t}^{i}\right\}_{i=1: m}$. The CGR algorithm iteratively updates the past belief $\operatorname{Bel}\left(x_{t-1}\right)$ using observation $y_{t}$ and control input $u_{t-1}$ as follows:

1) Samples of the belief $\operatorname{Bel}\left(x_{t-1}\right)$ are evolved through the motion model, $p\left(x_{t} \mid x_{t-1}, u_{t-1}\right)$ to generate a first stage proposal distribution $q^{0}$.

2) Samples of $q^{0}$ are "refined" in $r$ iterations (which produce intermediate distributions $q^{i}, i \in[1, r-i]$ ) using the gradients $\frac{\delta}{\delta x} p\left(y_{t} \mid x\right)$ of the observation model $p\left(y_{t} \mid x\right)$.

3) Samples of the last generation proposal distribution $q^{r}$ and the first stage proposal distribution $q^{0}$ are sampled using an acceptance test to generate the final proposal distribution $q$.

4) Samples $x_{t}^{i}$ of the final proposal distribution $q$ are weighted by corresponding importance weights $w_{t}^{i}$, and resampled with replacement to generate $\operatorname{Bel}\left(x_{t}\right)$.

Therefore, for CGR we need to compute both the observation likelihood, as well as its gradients. The observation likelihood is computed using Eq. 1, and the corresponding gradients are therefore given by,

$$
\frac{\delta}{\delta x} p(y \mid x)=-\frac{p(y \mid x)}{f \sigma^{2}} \sum_{i=1}^{n}\left[d_{i} \frac{\delta d_{i}}{\delta x}\right] .
$$

The term $\frac{\delta d_{i}}{\delta x}$ in this equation has two terms, corresponding to the translation component $\frac{\delta d_{i}}{\delta x_{1}}$ and the rotational component $\frac{\delta d_{i}}{\delta x_{2}}$. These terms are computed by rigid body translation and rotation of the point cloud $P$ respectively.

The observation likelihoods and their gradients thus computed are used to update the localization using CGR. 


\section{NAVIGATION}

For the robot to navigate autonomously, it needs to be able to successfully avoid obstacles in its environment. This is done by computing open path lengths available to the robot for different angular directions. Obstacle checks are performed using the 3D points from the sets $P$ and $O$. Let $r$ be the robot radius, $\theta_{d}$ the desired direction of travel, and $\hat{\theta}$ a unit vector in the direction of $\theta$. The open path length $d(\theta)$ and the chosen obstacle avoidance direction $\theta^{*}$ are hence calculated as:

$$
\begin{aligned}
P_{\theta} & =\{p: p \in P \cup O \wedge\|p-p \cdot \hat{\theta}\|<r\} \\
d(\theta) & =\min _{p \in P_{\theta}}(\max (0,\|p \cdot \hat{\theta}\|-r)) \\
\theta^{*} & =\arg \max _{\theta}\left(d(\theta) \cos \left(\theta-\theta_{d}\right)\right)
\end{aligned}
$$

\section{EXPERIMENTAL RESULTS}

We evaluated the performance of our depth camera based localization and navigation algorithms over two sets of experiments. The first set of experiments compare our approach using FSPF CGR localization to three other approaches that use the Kinect for localization by simulating laser rangefinder scans from the Kinect sensor. The second set of long run trials test the effectiveness of our complete localization and navigation system over a long period of time.

Our experiments were performed on our custom built omnidirectional indoor mobile robot, equipped with the Microsoft Kinect sensor. The Kinect sensor provides depth images of size $640 \times 480$ pixels at $30 \mathrm{~Hz}$. To compare the accuracy in localization of the different approaches using the Kinect, we also used a Hokuyo URG-04LX 2D laser rangefinder scanner as a reference. The autonomous long run trials were run using the Kinect alone for localization and navigation. All trials were run single threaded, on a single core of an Intel Core i7 950 processor.

\section{A. Comparison of FSPF to Simulated Laser Rangefinder localization}

We compared our approach using FSPF CGR localization to the following three other CGR based laser rangefinder localization algorithms where the data from the Kinect sensor was used to simulate laser rangefinder scans:

1) Extracting a single raster line from the Kinect depth image, reconstructing the corresponding $3 \mathrm{D}$ points, and then down-projecting into 2D to generate the simulated laser rangefinder scans. We call this approach the Kinect-Raster (KR) approach.

2) Randomly sampling locations in the Kinect depth image, and using the corresponding 3D points to simulate the laser rangefinder scan. We call this approach the Kinect-Sampling (KS) approach.

3) Reconstructing the full $3 \mathrm{D}$ point cloud from the entire Kinect depth image, and using all these points to generate the simulated laser rangefinder scan. We call this approach the Kinect-Complete $(\mathrm{KC})$ approach.

In each of these cases, we used simulated laser rangefinder readings with the CGR algorithm for localization, which has

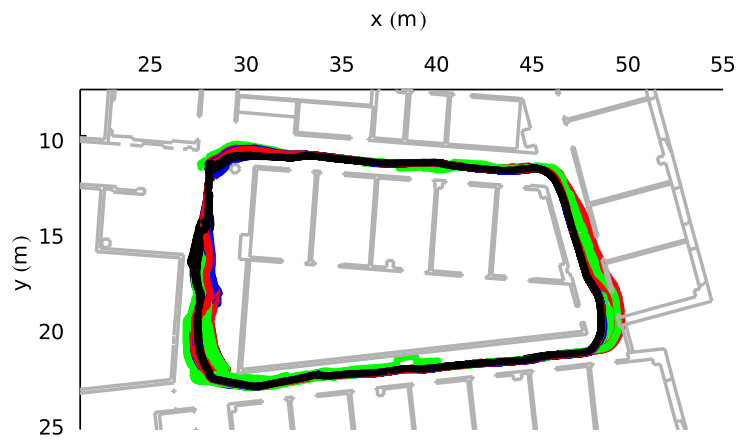

Fig. 5. Combined traces of all successful trials of all approaches: green $(\mathrm{KR})$, red $(\mathrm{KS})$, blue (KC) and black (FSPF). FSPF CGR localization is seen to have the least variation across trials.

been shown to be both robust as well as accurate when using a real laser rangefinder [15].

For estimating the error in localization using the Kinect, we used the localization estimates produced by the laser rangefinder CGR localization algorithm for reference. In the $\mathrm{KR}, \mathrm{KS}$ and $\mathrm{KC}$ approaches, the simulated laser rangefinder scan had a simulated angular range of $180^{\circ}$ and an angular resolution of $0.35^{\circ}$, although only part of this scan was populated with useful information due to the limited angular field of view of the Kinect. The number of points sampled in the KS approach was limited to 2000 points, the same as the number of plane filtered points generated by FSPF.

We recorded a log with odometry and data from the Kinect sensor and the laser rangefinder while traversing a path consisting of corridors as well as open areas with unmapped obstacles. There was significant human traffic in the environment, with some humans deliberately blocking the path of the robot. This log was replayed offline for the different localization approaches, running 100 times per approach, with randomly added $20 \%$ noise to the odometry data. Fig. 5 shows the combined traces of the localization estimates for all the succesful trials of each of the approaches. A trial was said to be "succesful" if the error in localization was less than $1 m$ at every timestep of the trial.

Fig. 7 shows a cumulative histogram of the error in localization using the different approaches. The FSPF approach has significantly less error than the other three approaches. Fig. 6 shows the cumulative failure rate as a function of the elapsed run time. The FSPF approach has a $2 \%$ failure rate at the end of all the trials, wehereas all the other approaches start failing after around $20 \mathrm{~s}$ into the trials. The $\mathrm{KR}, \mathrm{KS}$, and $\mathrm{KC}$ approaches have total failure rates of $82 \%, 62 \%$ and $61 \%$ respectively. The abrupt increase in failures around the $20 s$ mark corresponds to the time when the robot encounters unmapped objects in the form of humans, tables and chairs in an open area.

To compare the execution times, we kept track of the time taken to process the Kinect depth images (including disk access times), and calculated the ratio of these execution times to the duration of the trials. These values are indicative of the mean CPU processing load on the robot. The values for the different algorithms were $0.01 \%, 3.6 \%, 56.6 \%$ and $16.3 \%$ 


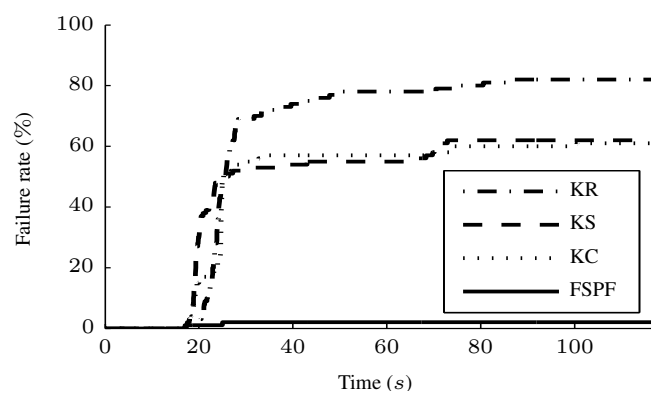

Fig. 6. Cumulative fractions of the failure rates as a function of elapsed time for all four approaches

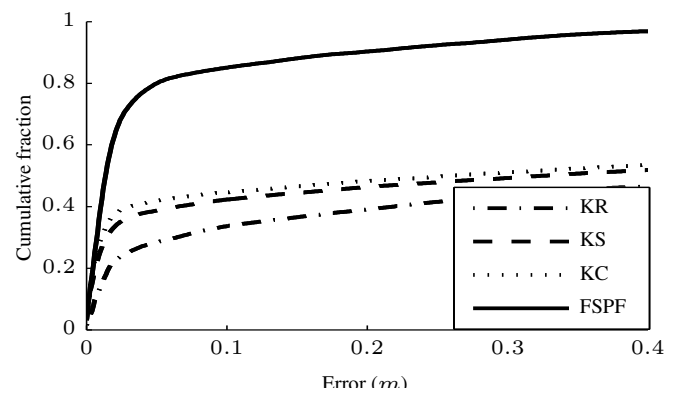

Fig. 7. Cumulative histogram of errors in localization of the different approaches. For each point $(x, y)$ on the curves, the $y$ value indicates the fraction of time that the algorithm had an error less than the $x$ value.

for the $\mathrm{KR}, \mathrm{KS}, \mathrm{KC}$, and $\mathrm{FSPF}$ approaches respectively.

\section{B. Long Run Trials}

To test the robustness of the depth-camera based FSPF localization and navigation solution, we set a series of random waypoints for the robot to navigate to, spread across the map. The total path had multiple passes over some corridors, and its length was just over $4 \mathrm{~km}$. Over the duration of the experiment, only the Kinect sensor was used for localization and obstacle avoidance. The robot successfully navigated to all waypoints, but localization had to be reset at three locations, which were in open areas of the map with unmapped obstacles where Kinect sensor could not observe any walls for a while. Fig. 8 shows the trace of the robot's location over the course of the experiment. We continue to use the FSPF localization and obstacle avoidance algorithms during daily deployments of our robot.

\section{CONCLUSiOn AND Future Work}

In this paper, we introduced an algorithm for efficient depth camera based localization and navigation for indoor mobile robots. We introduced the Fast Sampling Plane Filtering algorithm to filter depth images into point clouds corresponding to local planes. We subsequently contributed an observation model that matches the plane filtered points to lines in the existing 2D maps for localization. Both the plane filtered, as well as the outlier point clouds are further used for obstacle avoidance. We experimentally showed FSPF localization to be more accurate as well as more robust compared to localization using Kinect based simulated laser rangefinder readings. We further demonstrated a long run

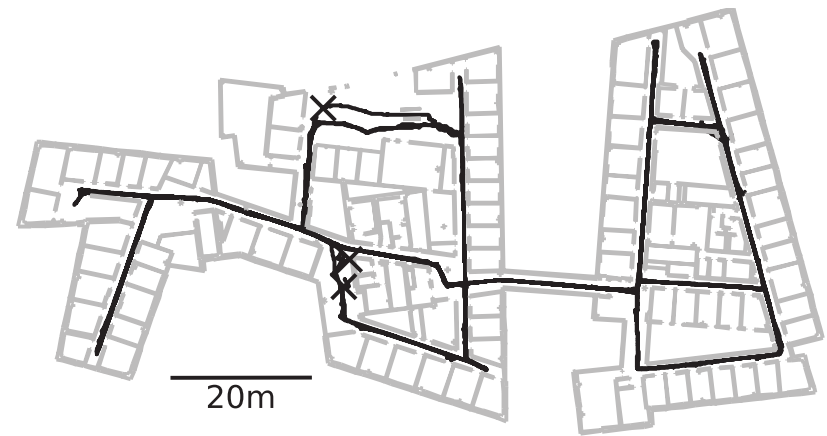

Fig. 8. Trace of robot location for the long run trial. The locations where localization had to be reset are marked with crosses.

trial of the robot autonomously operating for over $4 \mathrm{~km}$ using the depth camera alone.

For use on other platforms like UAVs, scaling up the state space to full 6 degrees of freedom is another possible avenue of future work.

\section{REFERENCES}

[1] N.J. Mitra and A. Nguyen. Estimating surface normals in noisy point cloud data. In Proceedings of the nineteenth annual symposium on Computational geometry, pages 322-328. ACM, 2003.

[2] R. Schnabel, R. Wahl, and R. Klein. Efficient RANSAC for PointCloud Shape Detection. In Computer Graphics Forum, volume 26, pages 214-226. Wiley Online Library, 2007.

[3] J. Poppinga, N. Vaskevicius, A. Birk, and K. Pathak. Fast plane detection and polygonalization in noisy $3 \mathrm{D}$ range images. In IROS 2008 , pages $3378-3383$.

[4] M.A. Fischler and R.C. Bolles. Random sample consensus: A paradigm for model fitting with applications to image analysis and automated cartography. Communications of the ACM, 24(6):381-395.

[5] H. Durrant-Whyte and T. Bailey. Simultaneous localization and mapping: part I. Robotics \& Automation Magazine, IEEE, 13(2):99110, 2006.

[6] D. Hähnel, W. Burgard, and S. Thrun. Learning compact 3D models of indoor and outdoor environments with a mobile robot. Robotics and Autonomous Systems, 44(1):15-27, 2003.

[7] A. Nuchter, K. Lingemann, J. Hertzberg, and H. Surmann. 6D SLAM with approximate data association. In Advanced Robotics, Proceedings of 12th International Conference on, pages 242-249. IEEE, 2005.

[8] A. Nüchter, K. Lingemann, J. Hertzberg, and H. Surmann. 6D SLAM - 3D mapping outdoor environments. Journal of Field Robotics, 24(89):699-722, 2007.

[9] J. Weingarten and R. Siegwart. 3D SLAM using planar segments. In IROS 2006, pages 3062-3067.

[10] P. Kohlhepp, P. Pozzo, M. Walther, and R. Dillmann. Sequential 3DSLAM for mobile action planning. In IROS 2004, pages 722-729.

[11] K. Pathak, A. Birk, N. Vaskevicius, M. Pfingsthorn, S. Schwertfeger, and J. Poppinga. Online three-dimensional SLAM by registration of large planar surface segments and closed-form pose-graph relaxation. Journal of Field Robotics, 27(1):52-84, 2010.

[12] P. Henry, M. Krainin, E. Herbst, X. Ren, and D. Fox. RGB-D mapping: Using depth cameras for dense $3 \mathrm{~d}$ modeling of indoor environments. In the 12th International Symposium on Experimental Robotics, 2010.

[13] A. Elfes. Using occupancy grids for mobile robot perception and navigation. Computer, 22(6):46-57, 1989.

[14] L. Zhang and B.K. Ghosh. Line segment based map building and localization using 2D laser rangefinder. In IEEE Int. Conf. on Robotics and Automation, 2000.

[15] J. Biswas, B. Coltin, and M. Veloso. Corrective gradient refinement for mobile robot localization. In IROS 2011.

[16] D. Fox, W. Burgard, F. Dellaert, and S. Thrun. Monte carlo localization: Efficient position estimation for mobile robots. In Proceedings of the National Conference on Artificial Intelligence, pages 343-349. JOHN WILEY \& SONS LTD, 1999. 